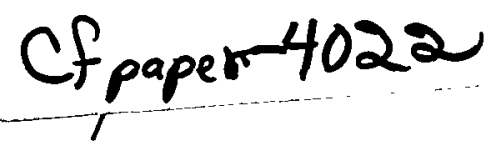

\title{
3-D Ray-tracing and 2-D Fokker-Planck Simulations of Radiofrequency Application to Tokamak Plasmas
}

\author{
F. PAOLETTI ${ }^{*}$, A. CARDINALI ${ }^{* *}$, S. BERNABEI ${ }^{* * *}$ \\ ${ }^{*}$ Columbia University, New York, NY, USA \\ ${ }^{* *}$ Associazione Euratom-ENEA sulla Fusione, Frascati, Rome, Italy \\ ${ }^{* * *}$ Plasma Physics Laboratory, Princeton, NJ, USA
}

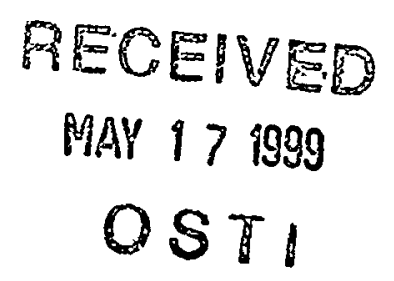

\begin{abstract}
A state of the art numerical tool has been developed to simulate the propagation and the absorption of coexisting different types of waves in a tokamak geometry. The code includes a numerical solution of the three-dimensional $(R, Z, \Phi)$ toroidal wave equation for the electric field of the different waves in the WKBJ approximation. At each step of integration, the two-dimensional $\left(v_{\|}, v_{\perp}\right)$ Fokker-Planck equation is solved in the presence of quasilinear diffusion coefficients. The electron Landau damping of the waves is modeled taking into account the interaction of the wave electric fields with the quasilinearly modified distribution function. Consistently, the code calculates the radial profiles of non-inductively generated current densities, the transmitted power traces and the total power damping curves. Synergistic effects among the different type of waves (e.g., lower hybrid and ion Bernstein waves) are studied through the separation of the contributions of the single wave from the effects due to their coexistence.
\end{abstract}

\section{INTRODUCTION.}

In the framework of the WKBJ (Wenzel-Kramers-Brillouin-Jeffrey) approximation for the geometric optics we developed a numerical code (QUASIRAY) that simulates propagation and absorption on the electron population of different types of waves in a toroidally axisymmetric geometry. The code is composed of three separate modules. A numerical equilibrium solver is used to provide the internal structure of the magnetic field on a rectangular mesh in $(R, Z)$ space. This equilibrium structure is used by the ray-tracing $(R T)$ module (1-3) to integrate a set of ordinary differential equations for the phase $S$ and the amplitude $A$ of the WKBJ electric field of the wave. The equilibrium is calculated on the ohmic plasma target and is not affected by the current drive i.e., modification of the distribution function (perturbative approach). The quasilinear modification of the distribution function due to the presence of the waves electric field is calculated at each step of the ray-tracing integration using the FokkerPlanck (FP) module (4-5). In the FP routine, a relativistic partial differential diffusive equation is solved in the 2-D velocity space $\left(v_{\|}, v_{\perp}\right)$. The 1-D spatial diffusion is neglected. In addition, we treat the steady state i.e., the distribution function does not depend on time and it is calculated balancing the collisional diffusion and the quasilinear diffusion due to the wave in the velocity space. The 


\section{DISCLAIMER}

This report was prepared as an account of work sponsored by an agency of the United States Government. Neither the United States Government nor any agency thereof, nor any of their employees, make any warranty, express or implied, or assumes any legal liability or responsibility for the accuracy, completeness, or usefuiness of any information, apparatus, product, or process disclosed, or represents that its use would not infringe privately owned rights. Reference herein to any specific commercial product, process, or service by trade name, trademark, manufacturer, or otherwise does not necessarily constitute or imply its endorsement, recommendation, or favoring by the United States Government or any agency thereof. The views and opinions of authors expressed herein do not necessarily state or reflect those of the United States Government or any agency thereof. 


\section{DISCLAIMER}

Portions of this document may be illegible in electronic image products. Images are produced from the best available original document. 
two-dimensional character of the FP solver allows the treatment of pitch-angle scattering of electrons in the velocity space.

\section{APPLICATION TO LHW+IBW SYNERGY SIMULATION.}

To illustrate the potentialities of the code, we applied it to the simulation of an experimental situation where lower hybrid wave (LHW) and Ion Bernstein wave (IBW) power is simultaneously applied in the electron-Landau damping regime. Experimental evidence of wave-wave synergy has been previously presented (6) and an exhaustive description/discussion of the details of a dedicated IBW+LHW experiment conducted on the PBX-M tokamak can be found in ref. (7). In this paragraph, we will concentrate on the results obtained using the QUASIRAY code. Preliminary studies on the LHW+IBW synergy with the QUASIRAY code were previously presented (8).

The plasma parameters of a typical target plasma used in the LHW+IBW experiment on PBX-M are: $\mathrm{I}_{\mathrm{p}}=120 \mathrm{kA}, \mathrm{B}_{0}=1.89 \mathrm{~T}, \mathrm{n}_{\mathrm{e}}(0)=2.510^{13} \mathrm{~cm}^{-3}, \mathrm{~T}_{\mathrm{e}}(0)=$ $1.1 \mathrm{keV}, \mathrm{R}_{\text {mag }}=164 \mathrm{~cm}, \mathrm{a}=32 \mathrm{~cm}, \mathrm{q}(0)=0.8, \mathrm{q}(\mathrm{a})=3.0$. IBWs were launched in the electron-damping regime with typical parameters: $v_{\mathrm{IBW}}=43 \mathrm{MHz}, \mathrm{n}_{\|}$(range) $= \pm[2-16], \mathrm{n}_{\|}$(peak) $= \pm 9, \mathrm{P}_{\mathrm{IBW}}=50 \mathrm{~kW}$, Pulse Duration $=400-45010^{-3} \mathrm{~s}$. The PBX-M LHW system was used to generate non-inductive current and its characteristics are: $v_{\mathrm{LH}}=4.6 \mathrm{GHz}, \mathrm{n}_{\|}($range $)=[1.9-2.3], \mathrm{n}_{\|}($peak $)=2.1, \mathrm{P}_{\mathrm{IBW}}=$ $139 \mathrm{~kW}$, Phase $=90^{\circ}$, Pulse Duration $=300-60010^{-3} \mathrm{~s}$. Different numerical scenarios have been considered to test the relative contribution of each of the two types of waves to the synergy process. In the different scenarios, we can either activate the quasilinear diffusion for that type of wave $D_{q l} \neq 0$ or else we can deactivate it $\left(D_{q 1} \neq 0\right)$ and consider only the linear damping $\left(D_{q 1}\right.$ is the quasilinear diffusion coefficient).

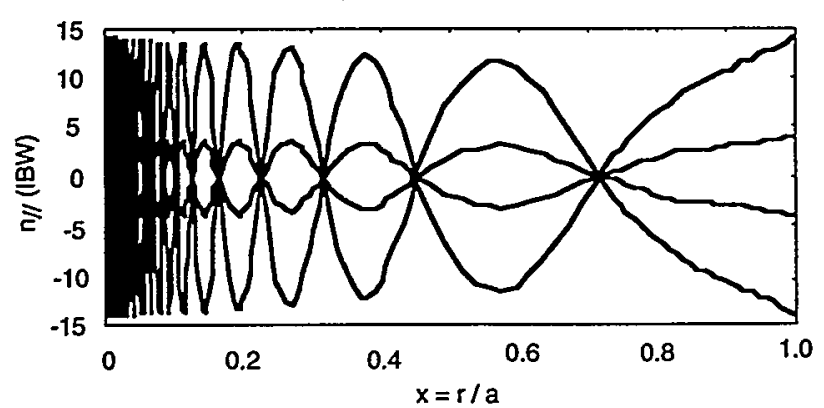

Figure 1. IBW $\mathrm{n} \|$ radial profile from RT calculations.
Since the IBW $\mathrm{n}_{\|}$is oscillating between positive and negative values as shown in Fig. 1, the modification of the distribution function takes place in the vicinities of the radial locations where $n_{\|}$reaches an extreme (maximum or minimum) i.e., where the wave is strongly

damped on the bulk of the distribution. Fig. 2 shows the distribution function at the location $x=0.56$ around the first IBW $n_{\|}$extreme. Since the IBW spectrum is symmetric, IBW alone is unable to generate a net non-inductive current but the quasilinear modifications to the distribution function can strongly affect the deposition of LHW power and the local current drive efficiency. During IBW injection (see Fig. 2), symmetric tails are produced in the $v_{j} / v_{\text {the }}$ space. In Fig. 2, we marked the $\mathrm{v}_{\|} / \mathrm{v}_{\text {the }}$ interval corresponding to the LHW spectrum. In the case of 
LHW injection alone, the lower extreme of the LHW $\mathrm{v}_{\|} / \mathrm{v}_{\text {the }}$ spectrum is intersecting the distribution function at a very low level $\left(\sim 10^{-6}\right)$.

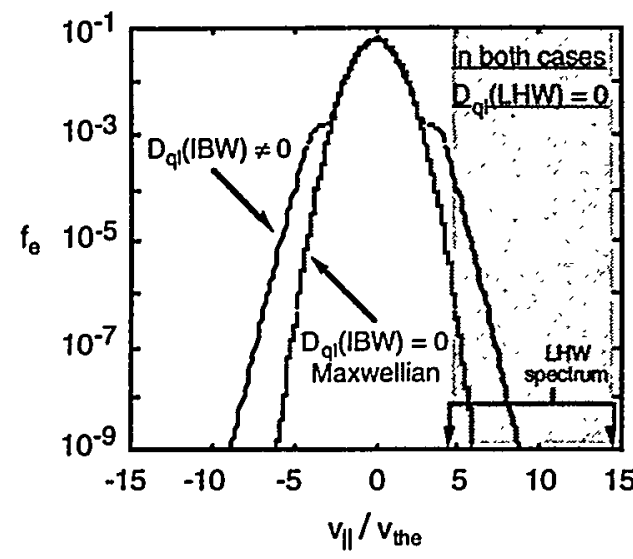

Figure 2. Maxwellian and quasi-linearly modified distribution functions during IBW injection at $x=0.56$. The shaded region is the LHW $\mathrm{v}_{\|} / \mathrm{v}_{\text {the }}$ interval at that location.

In this case, a very small number of electron is interacting with the wave and the amount of current generated is almost inappreciable. As IBW is turned on $\left(D_{\mathrm{q}}(\mathrm{IBW}) \neq 0\right.$ in Fig. 2), the lower extreme of the LHW $\mathrm{v}_{\|} / \mathrm{v}_{\text {the }}$ spectrum is intersecting a broader distribution function at a value that is roughly 2 orders of magnitude larger than the one we found in the previous case. Therefore, LHW can now generate a plateau in the distribution function which contains a number of nonthermal electrons that is 2 orders of magnitude larger than the one produced in the case of LHW injected alone. From the accounting of the LHW deposited power, we found that $20 \%$ of the total power carried by the wave is damped in the spatial interval $0.56<\mathrm{x}<1.0$ giving a high rate of transmitted power per single electron in that region of space which results in the formation of a localized current channel.

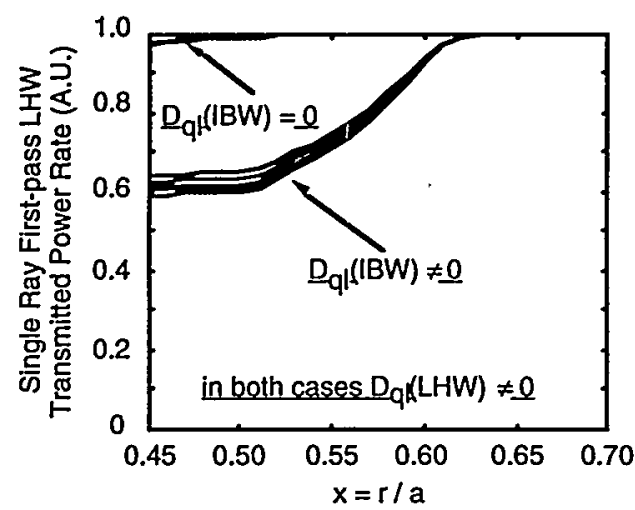

Figure 4. "First-pass" LHW transmitted power curves for five rays with values of $n_{\|}$ equi-spaced in the interval $[1.9,2.3]$

In the plasma regimes we are studying (low $\mathrm{T}_{\mathrm{e}}$ circular plasmas), the LHW is typically centrally deposited in the socalled "multi-pass" absorption scheme. The intrinsic validity of this scheme as well as the impact on the current drive localization capabilities are extensively discussed in refs. (9-11). As it is clear that in this condition localized off-axis LHCD cannot be obtained using LHW alone, during combined LHW+IBW injection the off-axis LHW damping is obtained at "first pass" as it is clearly shown in Fig.

4. This result ("first-pass" LHW damping on a tail) provides particular robustness to the model (WKBJ ray-tracing) and its linear treatment of the wave-wave interaction which could become questionable in the case of multiple wave reflections ("multi-pass" scheme) due to the possible existence of non-linear coupling.

The particular nature of the synergy between LHW and IBW (i.e., spatial localization of the non-inductive driven current) suggests its possible successful application to tearing mode stabilization (12). As described in detail in refs. (1213) the generation of a localized non-inductive current perturbation inside the 
magnetic island can stabilize the mode. Therefore, additional experimental investigations of the synergy process are needed. These further studies should be aimed at determining the level of accuracy to which the spatial location of the localized current channels can be varied as a function of certain plasma parameters (7), An ideal ground for this kind of systematic study is the FTU. tokamak where both RF systems are present and successfully funtioning (14-15).

\section{CONCLUSIONS.}

A multi-wave RT + FP algorithm (QUASIRAY) has been developed and tested on the modeling of LHW and IBW simultaneous application to plasmas confined in a toroidal magnetic geometry. The results of the study show a strong interaction between the two types of wave through their quasilinear modification of the distribution function. The model suggests the formation of localized non-inductive current channels during combined waves injection. Good agreement with the theoretical predictions has been found using experimental data from the PBX-M tokamak (comparison presented elsewhere). The specific characteristic of this multi-wave RF scheme suggests its possible application to non-inductive current drive stabilization of MHD tearing modes. In this regard, additional systematic experimental studies are required to assess the actual potentiality of this scheme with further validation of the theoretical predictions.

ACKNOWLEDGMENTS. We acknowledge L.M. Carlucci for her technical/editorial comments. Work supported by USDOE Contracts: DE-FG0289ER53297 and DE-AC02-76CH03073.

\section{REFERENCES.}

1 A. Cardinali, and F. Romanelli, Phys. Fluids B 4, 504 (1992).

2 A. Cardinali, Phys. Fluids B 5, 2778 (1993).

3 A. Cardinali, et al., Phys. Plasmas 2, 1510 (1995).

4 M. Shoucri and I. Shkarowsky, Comput. Phys. Commun. 78, 199 (1993).

5 M. Shoucri and I. Shkarowsky, Comput. Phys. Commun. 82, 287 (1994).

6 F. Paoletti, et al., Proceedings of the 12th Topical Conference on Radio Frequency Power in Plasmas, Savannah, GA, AIP Conf. Proc. 403, 1997, p. 173.

7 F. Paoletti, et al., Phys. Plasmas 6, 863 (1999).

8 F. Paoletti, et al., Proceedings of the 11th Topical Conference on Radio Frequency Power in Plasmas, Palm Springs, CA, AIP Conf. Proc. 355, 1995, p. 297.

9 F. Paoletti, et al., Nucl. Fusion 34, 771 (1994).

10 S. Bernabei, et al., Phys. Plasmas 4, 125 (1995).

11 Y. Peysson, "Status of Lower Hybrid Current Drive", in Proceedings of this Conference.

12 G. Giruzzi, et al., "Review of Tearing Mode Stabilization by RF Power in Tokamaks", in Proceedings of this Conference.

13 S. Bernabei, et al., Nucl Fusion 38, 87 (1998).

14 A. Tuccillo, et al., Proceedings of the 12th Topical Conference on Radio Frequency Power in Plasmas, Savannah, GA, AIP Conf. Proc. 403, 1997, p. 121.

15 R. Cesario, et al., "Recent Results of the Ion-Bernstein Wave Heating Experiment on FTU", in Proceedings of this Conference. 